\title{
RADIATION BOUNDARY CONDITIONS FOR THE TWO-DIMENSIONAL WAVE EQUATION FROM A VARIATIONAL PRINCIPLE
}

\author{
JAN BROEZE AND EDWIN F. G. VAN DAALEN
}

\begin{abstract}
A variational principle is used to derive a new radiation boundary condition for the two-dimensional wave equation. This boundary condition is obtained from an expression for the local energy flux velocity on the boundary in normal direction. The wellposedness of the wave equation with this boundary condition is analyzed by investigating the energy of the system. Results obtained with this (nonlinear) boundary condition are compared with those obtained with the (linear) first-order absorbing boundary condition suggested by Higdon.

In an accompanying paper the underlying theory is presented.
\end{abstract}

\section{INTRODUCTION}

Radiation (or absorbing) boundary conditions have the property that wave motions from the interior of the domain pass through the boundary with small reflections. Such boundary conditions are used to simulate artificial open boundaries, which may be introduced to reduce the computational domain.

Several methods to derive radiation boundary conditions for the two-dimensional wave equation are known.

Engquist and Majda [3, 4] have derived a perfectly absorbing boundary condition for plane waves travelling towards the boundary. However, this boundary condition is nonlocal in space and in time. Therefore, they developed a hierarchy of local boundary conditions, which approximate the perfectly absorbing boundary condition.

Higdon $[5,6]$ developed absorbing boundary conditions based on the dispersion relation for the discrete problem. His aim was to reduce the reflections for a priori known solutions as much as possible.

The boundary conditions developed by Bayliss and Turkel [1] are based on the behavior of the solution in the neighborhood of infinity.

The boundary conditions mentioned above have been developed for a certain class of solutions, i.e., the solution of the problem is assumed to have a certain

Received June 21, 1990; revised January 9, 1991.

1991 Mathematics Subject Classification. Primary 35L05, 35L65; Secondary 65M99, 65N99.

Key words and phrases. Absorbing boundary conditions, radiation boundary conditions, variational principle, conservation laws, wave equations, wellposedness.

These investigations were carried out under the supervision of Professor P. J. Zandbergen and Professor E. van Groesen (Twente University). The investigations were supported by the Netherlands Technology Foundation (STW) and by the Dutch Working-Group on Supercomputers (WGS). 
form beforehand. In this paper, radiation boundary conditions will not be derived under such assumptions, but by considering the energy transmission at the boundaries. The velocity of the energy on the boundaries in normal direction can be found by assuming the domain to be time-dependent. A radiation boundary condition is then found by substituting this velocity into the general boundary condition derived from the variational principle, as shown in [2].

It will be shown later on that the new boundary condition can be considered an extension of Higdon's first-order absorbing boundary condition. In Higdon's boundary conditions the absorption direction must be prescribed, whereas in our boundary condition the absorption direction is determined from the local solution near the boundary.

The outline of this paper is as follows. The variational principle will be applied to the problem in $\S 2$. In $\S 3$ we will present some boundary conditions which provide a well-posed problem. The quality of the boundary conditions for radiating waves will be analyzed in $\S 4$, and test results will be presented in $\S 5$.

\section{THE VARIATIONAL METHOD FOR THE TWO-DIMENSIONAL WAVE EQUATION}

In this section the variational method will be used to derive radiation boundary conditions for the two-dimensional wave equation.

In order to derive radiation boundary condition, we will first consider a timedependent domain $\Omega$, where the evolution of $\Omega$ is assumed to be given beforehand. Expressions for the velocity of the boundary of $\Omega$ will be given in the next section.

Consider the two-dimensional wave equation

$$
u_{t t}=\Delta u \text {, }
$$

where $\Delta$ is the Laplace operator in two dimensions $x$ and $y$. is

The action integral for this problem defined on a time-dependent domain $\Omega$

$$
I=\int_{T} \iint_{\Omega(t)} \frac{1}{2}\left(u_{t}^{2}-(\nabla u)^{2}\right) d \Omega d t
$$

where $T$ indicates a time interval and $\Omega(t)$ indicates the time-dependent domain in $x, y$-space.

A variation $\delta u$ of $u$ in (2.2) provides a variation in the action integral $I$ :

$$
\begin{aligned}
\delta_{u} I= & -\int_{T} \iint_{\Omega(t)}\left(u_{t t}-\Delta u\right) \delta u d \Omega d t \\
& +\int_{T} \frac{d}{d t}\left\{\iint_{\Omega(t)} u_{t} \delta u d \Omega\right\} d t-\int_{T} \int_{\partial \Omega}\left\{v_{n} u_{t}+u_{n}\right\} \delta u d S d t .
\end{aligned}
$$

In this equation, $v_{n}$ represents the velocity of the boundary in normal direction (the normal $\underline{n}$ is pointing outward).

The second integral in the right-hand side of (2.3) integrates out to the boundaries of the time interval $T$ and vanishes if $\delta u$ is assumed to vanish there. For the first variation of the action integral $I$ to vanish for any variation $\delta u, u$ must satisfy the wave equation (2.1) on the interior of the domain and the boundary condition

$$
u_{n}+v_{n} u_{t}=0 \text { on } \partial \Omega,
$$


or $u$ must be prescribed on the boundaries (which corresponds to $\delta u=0$ on the boundary $\partial \Omega$ ).

Note that no conditions are imposed on the velocity of the boundary. Obviously, there is a degree of freedom in the system. We will derive additional boundary conditions for $v_{n}$ in the next section.

We shall now investigate the wellposedness of (2.1) with boundary condition (2.4) on a boundary of a fixed domain, when the normal velocity does not necessarily vanish, simulating the boundary to be moving at that velocity. To this end, we will consider the energy integral given by

$$
E=\iint_{\Omega} \frac{1}{2}\left(u_{t}^{2}+(\nabla u)^{2}\right) d \Omega .
$$

Differentiation of equation (2.5) with respect to time gives

$$
\frac{d E}{d t}=\iint_{\Omega}\left\{u_{t t}-\nabla u\right\} u_{t} d \Omega+\int_{\partial \Omega} u_{n} u_{t} d S
$$

If $u$ satisfies the field equation, only energy outflow will occur if $u_{n} \cdot u_{t} \leq 0$ on the boundary. This corresponds to a positive $v_{n}$ in (2.4). Therefore, the problem is well posed (in the sense that the energy of the system does not increase) if (2.4) is used with $v_{n} \geq 0$.

Next consider the generation of waves on a boundary, by imposing an external force $f(x, y, t)$ on a part $S_{1}$ of the boundary $\partial \Omega$. The action integral for this problem is

$$
I=\int_{T} \iint_{\Omega(t)} \frac{1}{2}\left(u_{t}^{2}-(\nabla u)^{2}\right) d \Omega d t+\int_{T} \int_{S_{1}} f(x, y, t) u d S d t
$$

The first variation of $I$ vanishes for all variations $\delta u$, if $u$ satisfies (2.1) together with the boundary conditions

$$
\begin{array}{ll}
u_{n}+v_{n} u_{t}=f(x, y, t) & \text { on } S_{1}, \\
u_{n}+v_{n} u_{t}=0 & \text { on } \partial \Omega \backslash S_{1} .
\end{array}
$$

The right-hand side $f$ in $(2.8 \mathrm{a})$ can be used to generate waves on a boundary part $S_{1}$. For appropriate $v_{n} \geq 0$ this condition also radiates waves from the interior of the domain.

\section{EXPRESSIONS FOR THE BOUNDARY VELOCITY}

In the previous section we found the outflow boundary condition (2.4) for the two-dimensional wave equation, where $v_{n}$ must be positive for wellposedness of the problem. In the derivation of this boundary condition, the velocity $v_{n}$ was assumed to be given. In this section we shall find expressions for this velocity in such a way that the boundary radiates outgoing waves.

3.1. Boundary condition from conservation of energy and momentum. An expression for $v_{n}$ can be obtained by considering a time-dependent domain $\Omega(t)$ with constant energy or with constant momentum in a certain direction. First we shall consider the energy on a time-dependent domain $\Omega(t)$,

$$
E=\iint_{\Omega(t)} \frac{1}{2}\left(u_{t}^{2}+(\nabla u)^{2}\right) d \Omega \text {. }
$$


Differentiating equation (3.1) with respect to the time and applying Gauss' divergence theorem gives

$$
\begin{aligned}
\frac{d E}{d t}= & \iint_{\Omega(t)}\left\{u_{t t}-\Delta u\right\} u_{t} d \Omega \\
& +\int_{\partial \Omega}\left\{u_{n} u_{t}+\frac{1}{2} \tilde{v}_{n}\left(u_{t}^{2}+(\nabla u)^{2}\right)\right\} d S,
\end{aligned}
$$

where $\tilde{v}_{n}$ is the velocity of the boundary in normal direction. The total amount of energy is conserved if the integrand in the last integral of (3.2) vanishes on the boundary. An expression for $\tilde{v}_{n}$ in the derivatives of $u$ is found in this way, and by substituting this velocity for $v_{n}$ into (2.4), the following boundary condition is obtained (see also [2]):

$$
L v_{n}=\frac{1}{2}\left(u_{t}^{2}-(\nabla u)^{2}\right) v_{n}=0 .
$$

This equation is satisfied if we choose $v_{n}=\sqrt{u_{n}^{2} /(\nabla u)^{2}}$ (positive for wellposedness of the problem) in (2.4):

$$
u_{t}=-u_{n} \sqrt{(\nabla u)^{2} / u_{n}^{2}}
$$

In [2] it is shown that the same boundary condition is obtained from conservation of momentum in any direction on a time-dependent domain.

3.2. Higdon's boundary condition. The first-order absorbing boundary condition proposed by Higdon [5] is of the form (2.4), with $v_{n}=\cos (\alpha)$, where $\alpha$ is the angle of perfect absorption. Higdon's condition reads

$$
u_{t}=-\frac{1}{\cos (\alpha)} u_{n},
$$

where $|\alpha|<\pi / 2$ must hold for wellposedness of the problem (this can be derived from both our wellposedness theory and from Higdon's analysis).

\section{REFLECTION PROPERTIES OF THE BOUNDARY CONDITIONS}

In this section we investigate the reflection properties of the boundary conditions mentioned in the previous section.

First we investigate the reflection properties for one monochromatic wave.

Boundary condition (3.4) is exact for one monochromatic radiating wave from any direction, without providing reflections.

Boundary condition (3.5) perfectly radiates waves at an angle of incidence $\theta= \pm \alpha$ with the normal. For waves from other directions $\theta$, the reflection coefficient $R$ (the ratio of the amplitudes of the reflected wave and of the incident wave) is

$$
R=-\frac{\cos (\alpha)-\cos (\theta)}{\cos (\alpha)+\cos (\theta)}
$$

Next we investigate how the above boundary conditions radiate multiple waves.

Boundary condition (3.5) is linear, so the reflection that it provides for multiple waves is equal to the superposition of the reflections for the separate wave components. 
The reflections provided by the nonlinear condition (3.4) for multiple waves cannot be analyzed by considering the reflections for each radiating wave separately, because of nonlinear interactions in this boundary condition. No appropriate formulation has been found for the reflections. Therefore the quality of (3.4) for multiple waves will be investigated by tests in the next section.

\section{Test Results}

In this section we test the stability of the above-mentioned boundary conditions for the two-dimensional wave equation and discuss the reflections caused by the boundary conditions.

5.1. Test model. The tests will be carried out on a rectangular equidistant grid. In the interior grid points, the wave equation is imposed with three-point central discretizations for the second-order derivatives. In this way an explicit expression is obtained for the value of $u$ on a new time level.

Second-order three-point discretizations are chosen for the first spatial derivatives on the boundaries. In order to prevent numerical instabilities, it is advantageous to use an implicit time integration scheme for the boundary conditions. For boundary condition (2.4) this can be achieved by determining $v_{n}$ on the previous time level from the expression in $\S 3.1$,

$$
v_{n}=\sqrt{u_{n}^{2} /(\nabla u)^{2}} \text {. }
$$

This value can be substituted in equation (2.4), which can then be solved with an implicit scheme. However, Higdon [6] has shown that no instabilities are to be expected if the product of $v_{n}$ and the time step is small enough. For practical reasons, an explicit scheme is therefore used, as long as no instabilities occur.

A special treatment is necessary in the corner points, because no unique normal is defined there. Therefore, we define the normal to be directed along the bisection line of the adjacent boundaries.

5.2. Results on plane waves. Plane waves are simulated by imposing equation (2.8) on the boundaries. The waves can be generated on two boundaries with a nonzero function $f$ in the right-hand side of equation (2.8a), and we choose (2.8) on the other (radiating) boundaries.

Boundary conditions (3.4) and (3.5) are tested for plane waves travelling at an angle $\theta=\pi / 6$ and $\theta=\pi / 3$ with the $x$-axis, with exact solution

$$
u=\cos (\underline{k} \cdot \underline{x}-t),
$$

where $\underline{k}=(\cos (\theta), \sin (\theta))^{T}$. The tests were done with (5.2) as the initial condition at $t=0$.

The spatial domain is given by $[0,2 \pi] \times[0,2 \pi]$. The mesh sizes are $\Delta x=$ $\Delta y=\pi / 25$, and the time step is $\Delta t=\Delta x / 10$. For these values of $\Delta x, \Delta y$, and $\Delta t$, the discretization errors are less than $2 \%$ of the amplitude of the initial solution.

In boundary condition (3.5), for each boundary, $\alpha$ is chosen such that the boundary condition is exact for incident waves at an angle $\theta=\pi / 6$ with the $x$-axis.

The maximum error in the results for $\theta=\pi / 6$ after one period $(t=2 \pi)$ is only due to discretization errors, and is below $2 \%$. The maximum error for 


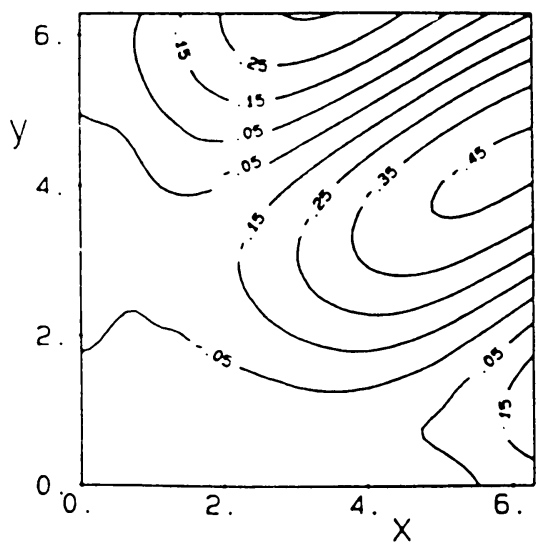

FIGURE 1

Errors after one period with boundary condition (3.5) for an incident wave (5.2) at $\theta=\pi / 3$

$\theta=\pi / 3$ with boundary condition (3.4) is also below $2 \%$, but (3.5) provides larger errors (over 45\%), as can be seen in Figure 1.

The errors obtained with (3.5) are larger than the reflection coefficient (4.1) indicates. This is due to the fact that no reflections are included in the initial solution.

Next we will test the radiation properties of these boundary conditions for a combination of two incident waves,

$$
u=A_{1} \cos \left(\underline{k}_{1} \cdot \underline{x}-t\right)+A_{2} \cos \left(\underline{k}_{2} \cdot \underline{x}-t+\beta\right),
$$

where $\underline{k}_{i}=\left(\cos \left(\theta_{i}\right), \sin \left(\theta_{i}\right)\right)^{T}$ and $\beta$ denotes a phase shift of the second wave component. The angles $\theta_{1}$ and $\theta_{2}$ are $\pi / 6$ and $\pi / 3$ in these tests.

The linear boundary condition (3.5) provides a reflection that is the superposition of the reflections obtained from each wave component in (5.3) separately (see $\S 4)$. That is why for $\beta=0$ the errors obtained with boundary condition (3.5) are the same as those in Figure 1.

In the previous section we concluded that the reflections obtained with (3.4) are not equal to the superposition of the reflections from each wave component separately. Since the interaction in the boundary condition between the incident wave components will depend on $\beta$, boundary condition (3.4) is tested for (5.3) with various choices of $\beta$. The errors in the results obtained after one period $(t=2 \pi)$ with boundary condition (3.4) for $\beta=0, \beta=\pi / 2$, and $\beta=\pi$ are given in Figures $2 a, 2 b$, and $2 c$, respectively.

The figures show that the errors in the results depend strongly on the phase shift $\beta$. The maximum errors for $\beta=0, \pi / 2$, and $\pi$ are $0.09,0.22$, and 0.34 , respectively.

In order to make a good comparison between the quality of the boundary conditions, the errors from the above tests are compared with those obtained with boundary condition (3.5), where $\alpha$ has an optimum value, i.e., $\alpha=\pi / 4$. The errors obtained with incident wave (5.3) with $\beta=\pi$ are shown in Figure 3 . The maximum error in this test is about 0.40 , which is larger than the maximum error obtained with boundary condition (3.4), as can be seen from Figures $2 \mathrm{c}$ and 3. 


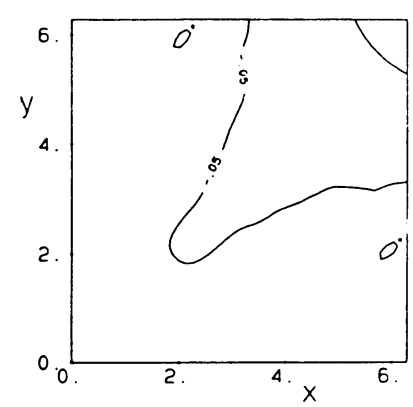

(a)

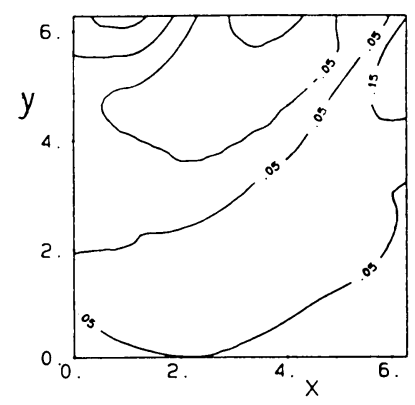

(b)

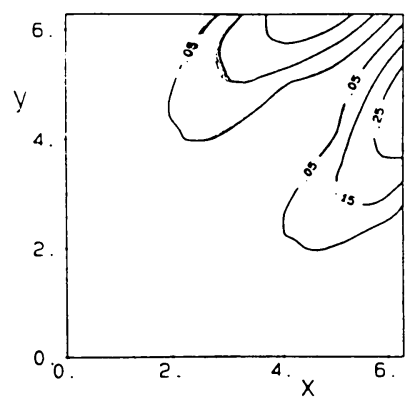

(c)

FIGURE 2

Errors after one period with boundary condition (3.4) for incident waves (5.3) with $A_{1}=A_{2}=1, \theta_{1}=\pi / 6, \theta_{2}=\pi / 3$, and $\beta=$ $0, \beta=\pi / 2$, and $\beta=\pi$, respectively. (a) (b) (c)

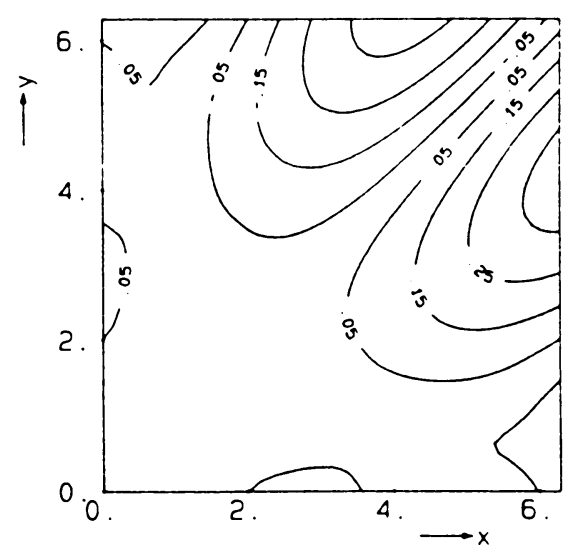

FIGURE 3

Errors after one period with boundary condition (3.5) for the incident wave (5.3) with $A_{1}=A_{2}=1, \theta_{1}=\pi / 6, \theta_{2}=\pi / 3$, and $\beta=\pi$.

We conclude that, in general, equation (3.4) provides smaller reflections than (3.5) does for plane waves. The reflections obtained with equation (3.4) depend on the interactions of multiple incident waves on the boundary, which is not the case for (3.5), but the maximum error obtained with (3.4) is smaller than the errors obtained with (3.5).

5.3. Reflections for an initial disturbance. In this subsection the quality of boundary conditions (3.4) and (3.5) will be tested numerically for an initial disturbance flowing out of the domain.

The problem is described in [5]. The initial disturbance is

$$
\begin{aligned}
u(x, y, 0) & = \begin{cases}\exp \left(-30 r^{2}\right) & (r<0.5), \\
0 & (r \geq 0.5),\end{cases} \\
u_{t}(x, y, 0) & =0,
\end{aligned}
$$

where $r^{2}=(x-0.5)^{2}+y^{2}$. As Higdon [5] notices, this disturbance is composed of Fourier modes corresponding to all possible directions of propagation. 
The solution to the wave equation will be computed on the domain

$$
\Omega_{1}=\{(x, y): 0<x<3,-2.5<y<2.5\},
$$

with boundary conditions (3.4) and (3.5) at $x=0$, respectively. The solution on the domain $\Omega_{1}$ will be compared with the solution on the larger domain

$$
\Omega_{2}=\{(x, y):-2<x<2,-2.5<y<2.5\} .
$$

The domains $\Omega_{1}$ and $\Omega_{2}$ are such that from $t=0$ until 2, reflections will occur only from the boundary conditions on $x=0$, and the solution on the larger domain $\Omega_{2}$ is used to measure the reflections from that boundary.

The errors in the computations with boundary conditions (3.4) and (3.5) (with $\alpha=0$ ) at $t=1.5$ are given in Figure 4.

The figures show that the errors in the solutions with boundary conditions (3.4) and (3.5) are of the same magnitude. Compared to (3.4), boundary condition (3.5) gives a poor representation of the solution near the boundary. This is due to the fact that in boundary condition (3.5) only normal derivatives (and no tangential derivatives) are included.

The development of the energy of the errors with boundary condition (3.4) and (3.5) (with $\alpha=0$ and $\alpha=\pi / 6$ ) is given in Figure 5. It can be seen that the energy of the errors obtained with (3.4) is smaller than the energy of the errors with (3.5), for $\alpha=0$ as well as for $\alpha=\pi / 6$. This is due to the fact that after a longer period the wave is travelling nearly tangentially to the boundary and is poorly simulated with boundary condition (3.5).

Imposing boundary condition (3.5) with $\alpha$ directed toward the center of the disturbance $(x, y)=(0.5,0)$ gives lower reflections than (3.4) does. However, this approach is only possible in simple problems where such details of the solution are known beforehand, whereas our condition has been developed for the situations when little is known about the solution of the system.

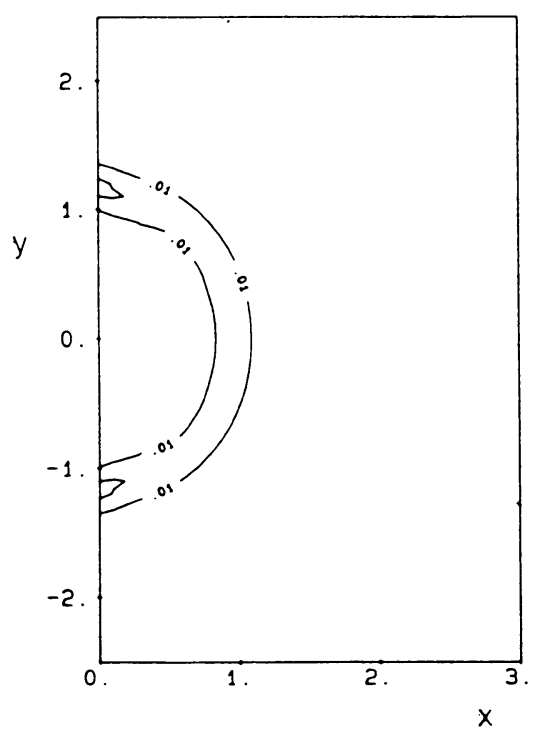

FIGURE 4a

Errors at $t=1.5$ with boundary condition (3.4) for initial disturbance (5.3) 


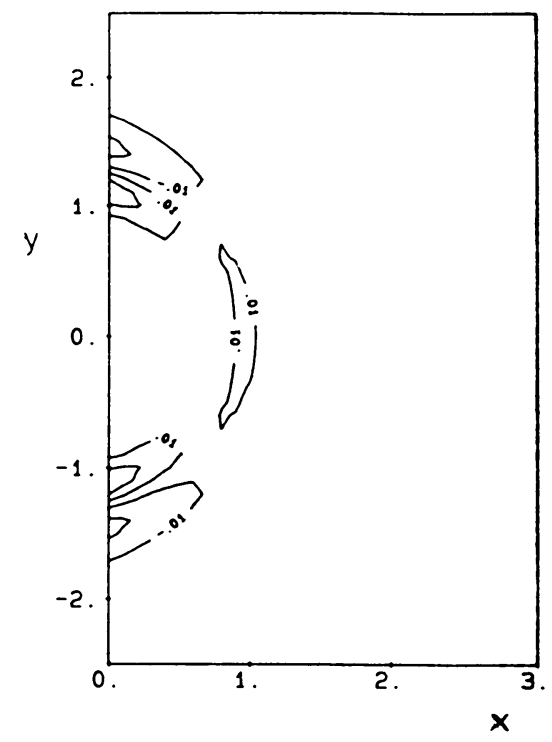

FIGURE 4b

Errors at $t=1.5$ with boundary condition (3.5) $(\alpha=0)$ for initial disturbance (5.3)

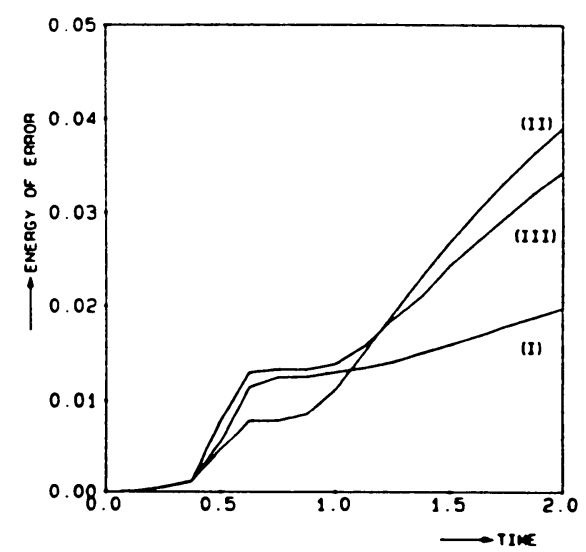

FIGURE 5

Energy of the reflections for incident wave (5.3); boundary condition (3.4): (I), boundary condition (3.5): (II) $(\alpha=0)$, (III) $(\alpha=\pi / 6)$.

We conclude that the solution obtained with boundary condition (3.4) is better than the solution obtained with (3.5) for the test case.

\section{Conclusions}

We conclude that the boundary conditions (3.4) and (3.5) provide reflections that are smaller than the incident waves. The new nonlinear boundary condition (3.4) can be used very well for radiating waves in one direction. The linear boundary condition (3.5) radiates waves travelling in a certain direction too, but the propagation direction must be known beforehand. The overall impression is that the boundary condition (3.4) provides lower reflections than (3.5) does. 


\section{BIBLIOGRAPHY}

1. A. Bayliss and E. Turkel, Far field boundary conditions for compressible flows, J. Comput. Phys. 48 (1982), 182-199.

2. E. F. G. van Daalen, J. Broeze, and E. van Groesen, Variational principles and conservation laws in the derivation of radiation boundary conditions for wave equations, Math. Comp. 58 (1992), 55-71.

3. B. Engquist and A. Majda, Absorbing boundary conditions for the numerical simulation of waves, Math. Comp. 31 (1977), 629-651.

4. _ Radiation boundary conditions for acoustic and elastic wave calculations, Comm. Pure Appl. Math. 32 (1979), 313-357.

5. R. L. Higdon, Absorbing boundary conditions for difference approximations to the multidimensional wave equation, Math. Comp. 47 (1986), 437-459.

6. _ Numerical absorbing boundary conditions for the wave equation, Math. Comp. 49 (1987), 65-90.

Delft Hydraulics, P.O. Box 152, 8300 AD Emmeloord, The Netherlands

Maritime Research Institute Netherlands, P.O. Box 28, 6700 AA Wageningen, The NETHERLANDS 